\title{
Single versus double row repair for full thickness rotator cuff tears using suture anchors. A systematic review and meta-analysis of basic biomechanical studies
}

Running Title: Basic Science Rotator Cuff Strength Meta-Analysis

\section{Erik Hohmann}

MBBS, FRCS, FRCS (Tr\&Orth), MD, PhD

Department of Orthopaedic Surgery, Clinical Medical School, University of Queensland, Australia

Faculty of Health Sciences, University of Pretoria, Pretoria, South Africa

\section{Anya König}

$\mathrm{BSc}$

Department of Anatomy, Faculty of Health Sciences, University of Pretoria, Pretoria, South Africa

\section{Cor-Jacques Kat}

$\mathrm{PhD}$

Faculty of Engineering, University of Pretoria, Pretoria, South Africa

\section{Vaida Glatt}

$\mathrm{PhD}$

University of Texas Health Science Center, San Antonio, Texas, USA

\section{Kevin Tetsworth}

MD, FRACS

Department of Orthopaedic Surgery, Royal Brisbane Hospital, Herston, Australia Department of Surgery, School of Medicine, University of Queensland, Australia Queensland University of Technology

Orthopaedic Research Institute of Australia 


\title{
Natalie Keough
}

$\mathrm{PhD}$

Department of Anatomy, Faculty of Health Sciences, University of Pretoria, Pretoria, South Africa

\section{Corresponding Author:}

Erik Hohmann

Department of Orthopaedic Surgery and Sports Medicine

Valiant Clinic/Houston Methodist Group

PO Box 414296

Dubai, United Arab Emirates

ehohmann@hotmail.com

\begin{abstract}
Purpose:

The purpose of this study was to perform a systematic review and meta-analysis comparing single- and double-row biomechanical studies to evaluate load to failure, mode of failure and gap formation.

\section{Materials and Methods:}

A systematic review of Medline, Embase, Scopus and Google Scholar was performed from 1990 through 2016. The inclusion criteria were: documentation of ultimate load to failure, failure modes, and documentation of elongation or gap formation. Studies were excluded if the study protocol did not use human specimens. Publication bias was assessed by funnel plot and Egger's test. The risk of bias was established using the Cochrane Collaboration's risk of bias tool. Heterogeneity was assessed using $\chi^{2}$ and $\mathrm{I}_{2}$ statistic.
\end{abstract}




\section{Results:}

Eight studies were included. The funnel plot was asymmetric suggesting publication bias, which was confirmed by Egger's test $(\mathrm{p}=0.04)$. The pooled estimate for load to failure demonstrated significant differences (SMD 1.228, 95\% CI: 0.55 to 5.226, $p=0.006, I_{2}=60.47 \%$ ), favouring double-row repair. There were no differences for failure modes. The pooled estimate for elongation/gap formation demonstrated significant differences ( $\mathrm{SMD} 0.783,95 \% \mathrm{CI}: 0.169$ to $1.398, \mathrm{p}=0.012, \mathrm{I}_{2}=58.8 \%$ ), favouring double-row repair.

\section{Conclusions:}

The results of this systematic review and meta-analysis suggest that double-row repair is able to tolerate a significantly greater load to failure. Gap formation was also significantly lower in the double-row repair group, but both of these findings should be interpreted with caution because of the inherent inter-study heterogeneity.

\section{Keywords:}

Basic science; biomechanics; rotator cuff tear; single row repair; double-row repair; meta-analysis; systematic review

\section{Level of evidence}

Systematic review and meta-analysis 


\section{Introduction}

Many factors contribute to successful repair of the rotator cuff. Anatomic factors are one of the key considerations, which include restoration of the footprint contact area [1], tendon and bone quality [2], appropriate compression of the tendon on the footprint to facilitate healing [3], and minimized motion at the bone-tendon interface [4]. Likewise, intrinsic factors such as decreased vascularity, hypoxia, atrophy, fibrocartilaginous changes, and fatty infiltration of the torn rotator cuff muscles have also been associated with inferior outcomes [2].

Initial fixation plays an important role and may be critical in achieving successful healing [2,5]. Numerous surgical techniques have been described for the repair of rotator cuff injuries, including fixation using single- or double-row suture anchor techniques, transosseous tunnels, or newer configurations such as transosseous equivalent (TOE) or knotless techniques [1]. Several biomechanical studies have demonstrated that double-row suture repair recreates the native footprint more closely resulting in significantly higher loads to failure and decreased gap formation under tensile loading [6-8]. In contrast, other basic science studies have not shown any significant differences between these two repair techniques [9-11].

Despite these results, debate continues as to whether double-row repair results in better clinical outcomes. A recent systematic review of overlapping meta-analyses suggested that double-row repair resulted in higher rates of structural healing when compared to single row repairs [12]. Unfortunately, these putative advantages do not seem to correlate with clinical observations. For example, a prospective randomized level 1 study by Burks et al. could not demonstrate any radiological or clinical 
differences between patients repaired with either single- or double-row techniques [13].

Given the lack of high level evidence from both clinical and basic science studies, the purpose here was to perform a systematic review and meta-analysis comparing singleto double-row biomechanical studies with respect to load to failure, mode of failure, and gap formation. We hypothesized that there would be no differences between these two techniques.

\section{Methods}

The research was conducted according to the methods described in the Cochrane Handbook [14]. The results are reported according to the Preferred Reporting Items for Systematic Reviews and Meta-Analysis (PRISMA) guidelines statement [15].

\section{Eligibility criteria}

Ex vivo basic science and biomechanical published studies that compared single- to double-row suture anchor techniques (Figure 1 and 2) between January 1990 and November 2016 were identified, and included if they fulfilled the following inclusion criteria: complete documentation of ultimate load to failure including standard deviation (SD) or range, description of failure mode for all specimens and documentation of elongation or gap formation including SD or range; complete description of the biomechanical testing set-up, surgical techniques, and methodology in human cadaveric studies. Studies comparing the more contemporary suture bridge or transosseous-equivalent (TOE) rotator cuff repair to single-row repairs were also included. Park, et al. demonstrated that both techniques were similar with the exception of ultimate load, which was higher in the TOE group $[16,17]$. 
Basic Science Rotator Cuff Strength Meta-Analysis

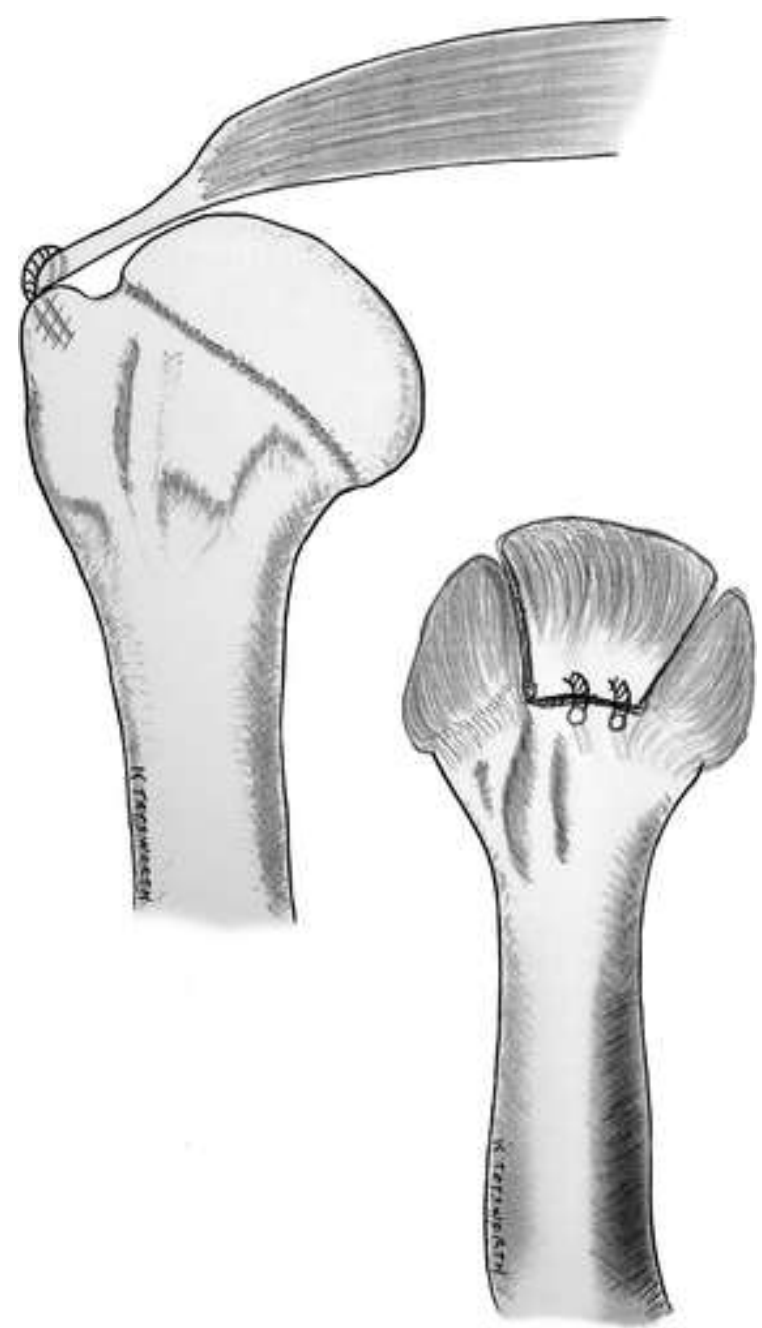

Figure 1: Single-row supraspinatus repair with two anchors placed at the lateral edge of the footprint 


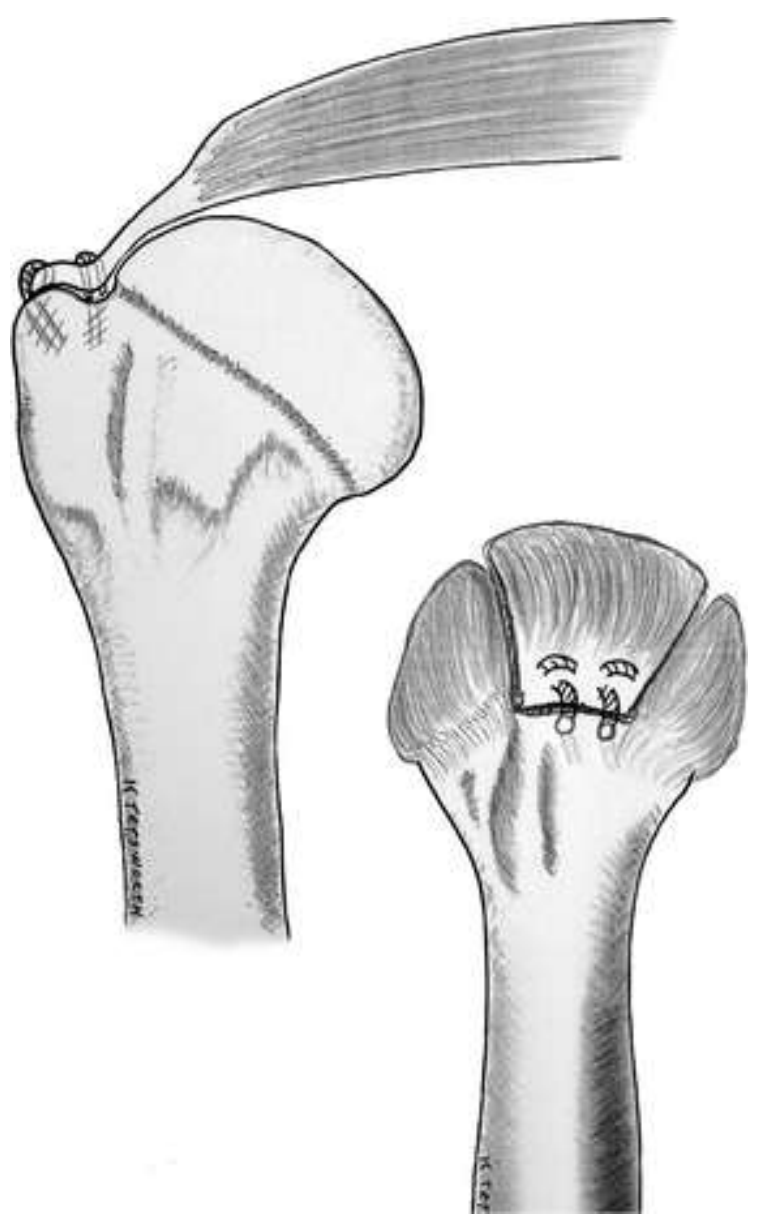

Figure 2: Double-row supraspinatus repair with a medial row typically placed just lateral to the articular margin and the lateral row placed just medial to the drop of the greater tuberosity.

Studies were excluded if the study protocol used ovine, bovine or other non-human specimens. Pietschmann, et al. demonstrated that ultimate load and primary stability of suture anchors under load depends mainly on cortical thickness and subcortical trabecular bone quality; hence, bovine and ovine humeri were deemed unsuitable for testing [18]. Furthermore, clinical outcome studies, research theses, conference proceedings or abstracts, and articles comparing other surgical techniques such as the transosseous Millett technique, or studies that have tested a combination of either one medial and two lateral, or two medial and one lateral anchor, were also excluded. 


\section{Literature research}

A systematic review of the literature was performed to identify all publications in the English and German literature dealing with biomechanical evaluation of rotator cuff repair. The databases Medline, Embase, Scopus and Google Scholar were systemically searched using the terms and Boolean operators: "rotator cuff" AND "biomechanical" AND/OR "cadaveric/cadaver"; "suture anchor" AND "arthroscopic" AND/OR "open". Three reviewers conducted independent title and abstract screening. All eligible articles were manually cross-referenced to ensure that other potential studies were included. Disagreements were resolved by consensus; if no consensus was achieved they were carried forward to the full text review, and then resolved by consensus. The search was performed on 5 November 2016.

\section{Data extraction and quality assessment}

For studies that met the inclusion criteria, an electronic data extraction form was used to obtain the following information from each article: author, journal and year of publication, any conflicts of interest, surgical technique, sample size, type of suture anchor and suture material, biomechanical testing details such as cyclic loading conditions, load to failure testing, failure modes, ultimate load to failure and SD, stiffness and SD, elongation and SD. Two authors independently completed data extraction, and the third reviewer and senior author verified the data.

Risk of bias was assessed adapting the Cochrane Collaboration's Risk of Bias Tool [14]. Cochrane's Handbook does not describe specifically the assessment of risk of bias for basic science and biomechanical studies, and the assessment tool described in chapter 8 addresses clinical studies only. The first four assessment items (random 
sequence generation, allocation concealment, blinding of participants and personnel, and blinding of outcome assessment) specifically relate to clinical studies only, and were not utilized in the analysis. Therefore, risk of bias assessment for this metaanalysis consisted only of the following three items: incomplete outcome data, selective reporting and other bias. Assessment of these three items was based on the recommendations by Hartling et al., as described in appendix B of their publication [19]. According to Hartling et al. and the recommendations in the Cochrane Handbook the risk of bias was categorized as 'low risk', 'unclear risk' and 'high risk' $[14,19]$.

The GRADE system was used by the senior author to assess the quality of the evidence for each outcome measure; the second reviewer verified the assessments. The recommendations from the Cochrane Handbook were followed and studies were downgraded if there were limitations in the design, indirectness of evidence, unexplained heterogeneity, imprecision of results, and high probability of publication bias. All institutional and author information was concealed to the second reviewer to reduce reviewer bias. Any disagreement between reviewers was resolved by a consensus and/or by arbitration between the two senior authors.

\section{Statistical analysis}

Inter-observer differences for study eligibility and risk of bias were measured using Cohen's kappa coefficient. Heterogeneity of the data was assessed using $\chi^{2}$ and $\mathrm{I}_{2}$ statistics. Outcomes were pooled using a fixed effect model if the $\mathrm{I}_{2}$ statistic was $<25 \%$; however, if it was $>25 \%$ then a random effect model was utilized. If standard deviations were not reported, the standard deviation was calculated using the 
following formula: $\mathrm{SD}=\max -\mathrm{min} / 4$. Hozo, et al. showed that this formula provides a good estimation for standard deviation [20]. The results of the categorical outcome recurrence were pooled and analysed using a contingency table and a Chi-Square test, or Fisher's exact test with Yates corrections if the sample size in any category was $<5$. All tests of significance were two-tailed, and an $\alpha$ of less than 0.05 was considered significant. Publication bias was assessed using funnel plots. If publication bias was present, Egger's test of intercept was performed to test for asymmetry. Funnel and forest plots, as well as all statistical analyses, were performed using STATA SE (Version 12.0; StataCorp, College Station, Texas, USA) for Windows and the comprehensive meta-analysis software package (CMA), version 3 (Biostat Inc, Englewood, NJ, USA).

\section{Results}

\section{Study selection and characteristics}

The literature search identified 128 studies for consideration; however, 78 studies were excluded following abstract screening. For the remaining studies, examination of the full text manuscripts was conducted, and only eight of the 50 articles met all eligibility criteria and were included in the analysis. (Figure 3) [7,8,10,11,21-24]. Overall, agreement between the two reviewers regarding final eligibility was excellent (kappa value 0.87, 95\% CI 0.82-0.90). All eight studies were published in English between 2006 and 2015, with a cumulative total of 106 specimens tested. The study characteristics and results are summarized in Tables 1 and 2. 
Basic Science Rotator Cuff Strength Meta-Analysis

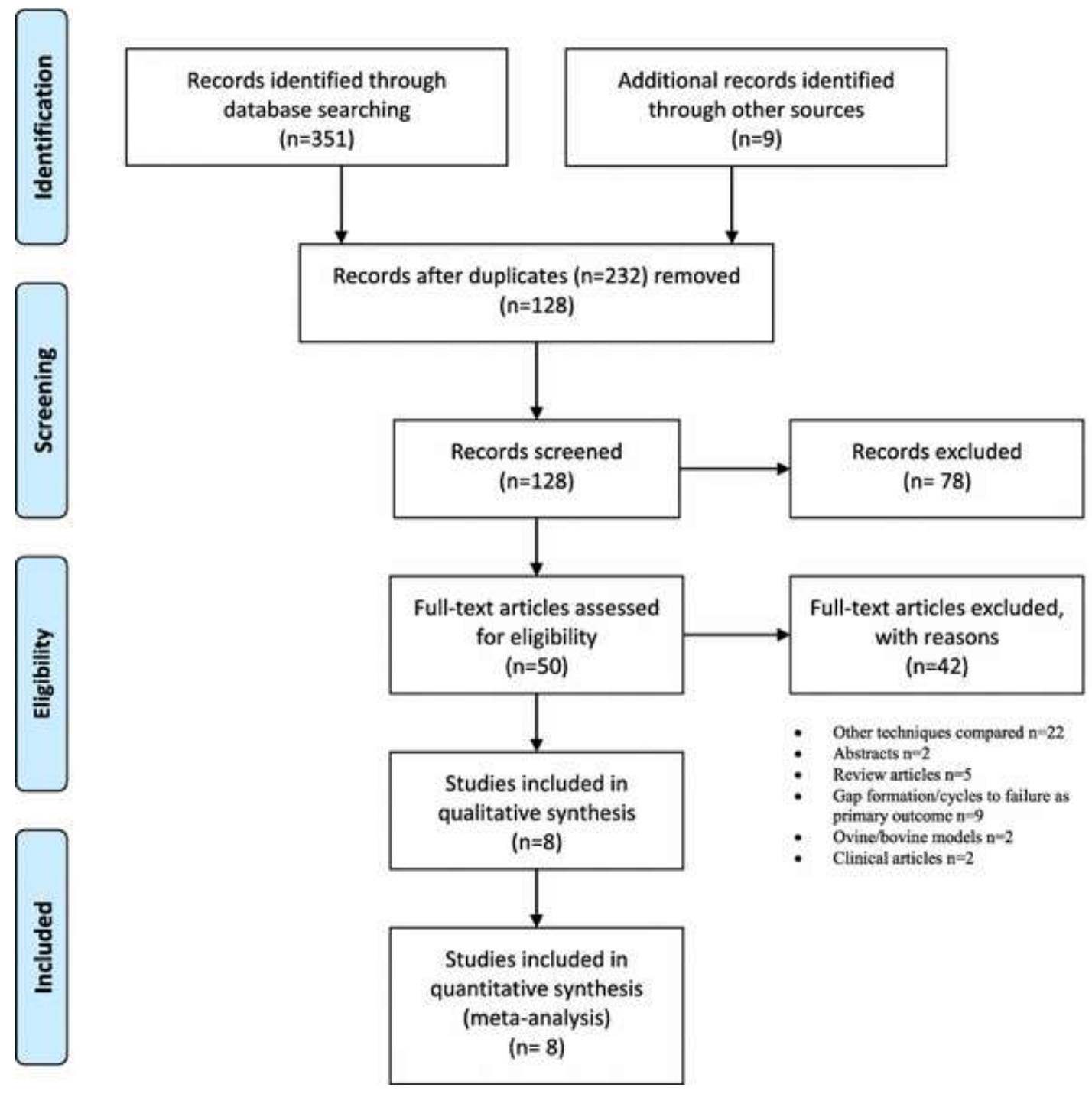

Figure 3: PRISMA Flow Diagram. From the initial 360 records, 8 studies were included. 
Table 1: Characteristics of the included studies

\begin{tabular}{|c|c|c|c|}
\hline Study & Study Design & $\begin{array}{c}\text { Sample } \\
\text { Size }\end{array}$ & Test Mode \\
\hline Mazzocca et al.; 2005 & $\begin{array}{l}\text { SR: } 3 \times 5 \mathrm{~mm} \text { Bio Corkscrews, single loaded \#2 Fibrewire } \\
\text { DR: Millett technique, } 2 \times \text { medial + lateral } 5 \mathrm{~mm} \text { Bio Corkscrews }\end{array}$ & 10 & $\begin{array}{l}3000 \text { cycles to } 100 \mathrm{~N} @ 1 \mathrm{~Hz} \\
\text { Load to failure @ } 31 \mathrm{~mm} / \mathrm{min} \\
\text { Humerus Position: N/A }\end{array}$ \\
\hline Smith et al.; 2006 & $\begin{array}{l}\text { SR: } 2 \times 5.0 \mathrm{~mm} \text { Twinfix, double loaded \#2 Durabraid } \\
\text { DR: } 4 \times 5.0 \mathrm{~mm} \text { Twinfix double loaded \#2 Durabraid }\end{array}$ & 16 & $\begin{array}{l}\text { Preload } 40 \mathrm{~N}, 10 \text { cycles } 40-50 \mathrm{~N} @ 50 \mathrm{~mm} / \mathrm{min} \\
10 \text { cycles to } 100 \mathrm{~N} \text {, increase by } 50 \mathrm{~N} \text { every } 10 \text { cycles until failure } \\
\text { Humerus Position @ } 30 \text { deg abduction }\end{array}$ \\
\hline Kim et al.; 2006 & $\begin{array}{l}\text { SR: } 2 \times 6.5 \mathrm{~mm} \text { Corkscrews, double loaded \#2 Fibrewire } \\
\text { DR: } 2 \times 6.5 \mathrm{~mm} \text { Corkscrews, double loaded \#2 Fibrewire }\end{array}$ & 18 & $\begin{array}{l}\text { Preload 10N, } 200 \text { cycles } 10-180 \mathrm{~N} @ 5 \mathrm{~Hz} \\
\text { Load to failure @ } 1 \mathrm{~mm} / \mathrm{sec} \\
\text { Humerus Position @ } 45 \mathrm{deg} \text { abduction }\end{array}$ \\
\hline Ma et al.; 2006 & $\begin{array}{l}\text { SR: } 5 \mathrm{~mm} \text { Corkscrew,\#2 Fibrewire, } \mathrm{n}=\text { ?? } \\
\text { DR: } 5 \mathrm{~mm} \text { Corkscrew,\#2 Fibrewire, } \mathrm{n}=? ?\end{array}$ & 10 & $\begin{array}{l}\text { Preload } 5 \mathrm{~N}, 50 \text { cycles } 5-100 \mathrm{~N} @ 0.25 \mathrm{~Hz} \\
\text { Load to failure @ } 1 \mathrm{~mm} / \mathrm{sec} \\
\text { Humerus Position @ } 45 \mathrm{deg} \text { abduction }\end{array}$ \\
\hline Domb et al.; 2008 & $\begin{array}{l}\text { SR: } 3 \times 6.5 \mathrm{~mm} \text { Corkscrews, double loaded \#2 Fibrewire } \\
\text { DR: } 3 \times \text { medial+lateral } 6.5 \mathrm{~mm} \text { Corkscrews, double loaded \# Fiberwire }\end{array}$ & 20 & $\begin{array}{l}\text { SR: } 200 \text { cycles } 60-230 \mathrm{~N} @ 5 \mathrm{~Hz} \\
\text { DR: } 200 \text { cycles } 10-180 \mathrm{~N} @ 5 \mathrm{~Hz} \\
\text { Load to failure @ } 1 \mathrm{~mm} / \mathrm{sec} \\
\text { Hummerus Position @ } 30 \mathrm{deg} \\
\end{array}$ \\
\hline Lorbach et al.; 2012 & $\begin{array}{l}\text { SR; } 2 \times 5.5 \text { Corkscrews, triple loaded \#2 Fibrewire } \\
\text { DR: } 2 \times 5.5 \text { Corkscrews med }+2 \times \text { Pushlock }\end{array}$ & 12 & $\begin{array}{l}\text { Preload } 40 \mathrm{~N}, 50 \text { cycles } 10-60 \mathrm{~N} \text {, increase to } 100,180,250 \mathrm{~N} \text { every } 50 \\
\text { cycles @33mm/sec } \\
\text { Load to failure @ ?? } \\
\text { Humerus Position @ ?? }\end{array}$ \\
\hline Barber et al.; 2012 & $\begin{array}{l}\text { SR: } 2 \times 5.5 \text { Corkscrews, triple loaded \#2 Fibrewire } \\
\text { DR: } 4 \times 5.5 \text { Swivelock, double loaded Fibretape }\end{array}$ & 18 & $\begin{array}{l}\text { Preload ?, } 200 \text { cycles } 10-100 \mathrm{~N} @ 1 \mathrm{~Hz} \\
\text { Load to failure @ } 33 \mathrm{~mm} / \mathrm{sec} \\
\text { Humerus Position @ } 45 \mathrm{deg} \text { abduction }\end{array}$ \\
\hline Esquivel et al.; 2015 & $\begin{array}{l}\text { SR: } 2 \times 5.5 \mathrm{~mm} \text { Corkscrews, double loaded \#2 Fibrewire } \\
\text { DR: } 2 \text { medial } 5.5 \text { Corkscrew+ } 2 \times \text { Pushlocks, double loaded \#2 Fibrewire }\end{array}$ & 10 & $\begin{array}{l}\text { Preload 2N } \\
\text { Load to failure @ } 1 \mathrm{~mm} / \mathrm{sec} \\
\text { Humerus Position @ } 45 \mathrm{deg} \text { abduction }\end{array}$ \\
\hline
\end{tabular}

Table 2: Characteristics of the included studies

\begin{tabular}{|c|c|c|c|c|c|c|}
\hline Study & Sample Size & Load to Failure & Stiffness & Elongation/Gap & $\begin{array}{l}\text { Failure Mode } \\
\text { Anchor } \\
\text { pullout/failure }\end{array}$ & $\begin{array}{l}\text { Failure Mode } \\
\text { Suture through } \\
\text { tendon }\end{array}$ \\
\hline Mazzocca et al.; 2005 & 10 & $\begin{array}{l}\text { SR: } 287.2+27.3 \\
\text { DR: } 256 .+27.8\end{array}$ & & $\begin{array}{l}\text { SR: } 2.28+0.26 \\
\text { DR: } 2.35+1.25\end{array}$ & $\begin{array}{l}\text { SR } 3 \\
\text { DR } 0\end{array}$ & $\begin{array}{l}\mathrm{SR} 2 \\
\mathrm{DR} 5\end{array}$ \\
\hline Smith et al.; 2006 & 16 & $\begin{array}{l}\text { SR: } 224+147.9 \\
\text { DR: } 320+96\end{array}$ & & $\begin{array}{l}\text { SR: } 5+1.2 \\
\text { DR: } 3.8+1.4\end{array}$ & $\begin{array}{l}\text { SR } 1 \\
\text { DR } 0\end{array}$ & $\begin{array}{l}\text { SR } 4 \\
\text { DR } 8\end{array}$ \\
\hline Kim et al.; 2006 & 18 & $\begin{array}{l}\text { SR: } 349+75.1 \\
\text { DR: } 516.3+120.8\end{array}$ & $\begin{array}{l}\text { SR: } 81.3+22.6 \\
\text { DR: } 118.4+15\end{array}$ & $\begin{array}{l}\text { SR: } 7.6+3.7 \\
\text { DR: } 3.6+2.6\end{array}$ & $\begin{array}{l}\text { SR: } 2 \\
\text { DR: } 2\end{array}$ & $\begin{array}{l}\text { SR:7 } \\
\text { DR: } 7\end{array}$ \\
\hline Ma et al.; 2006 & 10 & $\begin{array}{l}\text { SR: } 191+18 \\
\text { DR: } 287+24\end{array}$ & $\begin{array}{l}\text { SR: } 93+6 \\
\text { DR: } 112+14\end{array}$ & $\begin{array}{l}\text { SR: } 1.4+0.2 \\
\text { DR: } 1.1+0.1\end{array}$ & $\begin{array}{l}\text { SR: } 0 \\
\text { DR: } 1\end{array}$ & $\begin{array}{l}\text { SR: } 5 \\
\text { DR: } 4\end{array}$ \\
\hline Domb et al.; 2008 & 20 & $\begin{array}{l}\text { SR: } 392.3+40 \\
\text { DR: } 644+65\end{array}$ & $\begin{array}{l}\text { SR: } 127.1+12 \\
\text { DR: } 202.3+20\end{array}$ & $\begin{array}{l}\text { SR: } 8.7 \\
\text { DR: } 5.6\end{array}$ & $\begin{array}{l}\text { SR: } 2 \\
\text { DR: } 1\end{array}$ & $\begin{array}{l}\text { SR: } 8 \\
\text { DR: } 7\end{array}$ \\
\hline Lorbach et al.; 2012 & 12 & $\begin{array}{l}\text { SR: } 532.8+106.7 \\
\text { DR: } 681+249.7\end{array}$ & & $\begin{array}{l}\text { SR: } 6.18+1.41 \\
\text { DR: } 7.26+2.97\end{array}$ & Not reported & \\
\hline Barber et al.; 2012 & 18 & $\begin{array}{l}\text { SR: } 393+118 \\
\text { DR: } 586+128\end{array}$ & & $\begin{array}{l}\text { SR: } 4.2+1.5 \\
\text { DR: } 2.5+0,6\end{array}$ & $\begin{array}{l}\text { SR: } 5 \\
\text { DR: } 7\end{array}$ & $\begin{array}{l}\text { SR: } 4 \\
\text { DR: } 2\end{array}$ \\
\hline Esquivel et al.; 2015 & 10 & $\begin{array}{l}\text { SR: } 309.5+129.8 \\
\text { DR: } 350.7+126\end{array}$ & $\begin{array}{l}\text { SR: } 13.6+9 \\
\text { DR: } 20.5+6.9\end{array}$ & $\begin{array}{l}\text { SR: } 28.4+4.9 \\
\text { DR: } 25.9+3\end{array}$ & $\begin{array}{l}\text { SR:2 } \\
\text { DR: } 2\end{array}$ & $\begin{array}{l}\text { SR: } 3 \\
\text { DR: } 3\end{array}$ \\
\hline
\end{tabular}

\section{Risk of bias}

The findings of the risk assessment bias are summarized in Table 3. Of the eight studies, only one study, by Lorbach et al. [24], was found to have a high risk of bias. The authors failed to report details for load to failure testing and humerus position during testing. Failure modes were also not included in their publication. Additionally, an unclear risk of bias was assessed in the study by Barber et al. where the preload was mentioned, but not its magnitude [21]. While the preload could be assumed to be $10 \mathrm{~N}$ based on the reported cyclic loads, it was not clear whether the 
initial cyclic loading force was equivalent to the preload (Table 1). Visual inspection of the funnel plot was clearly asymmetric, suggesting publication bias (Figure 4). Moreover, Egger's intercept value was significant ( $\mathrm{p}=0.04$ two-tailed) and was calculated to be 7.5 (95\% CI: $0.42-14.48, \mathrm{t}=2.59)$, confirming publication bias was present.

Table 3: Risk of bias

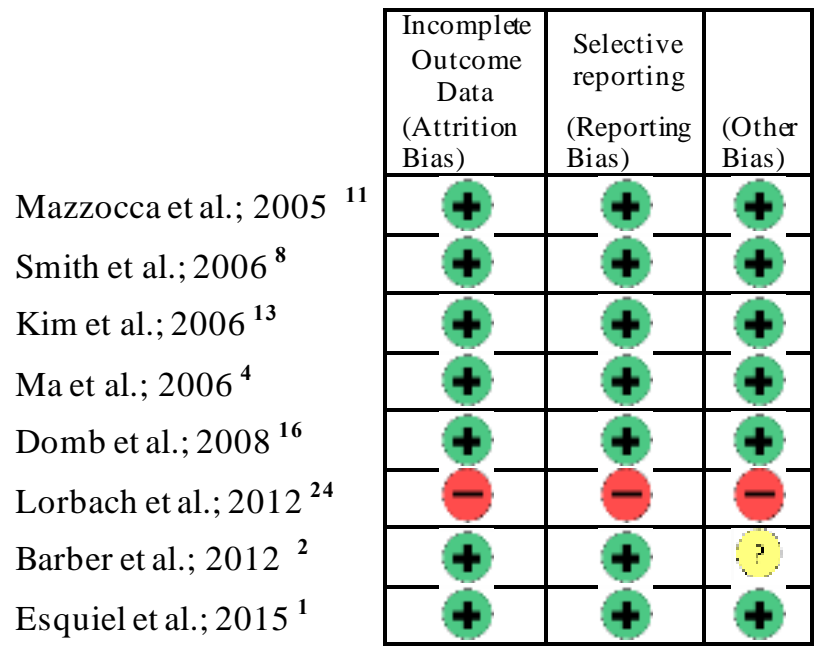




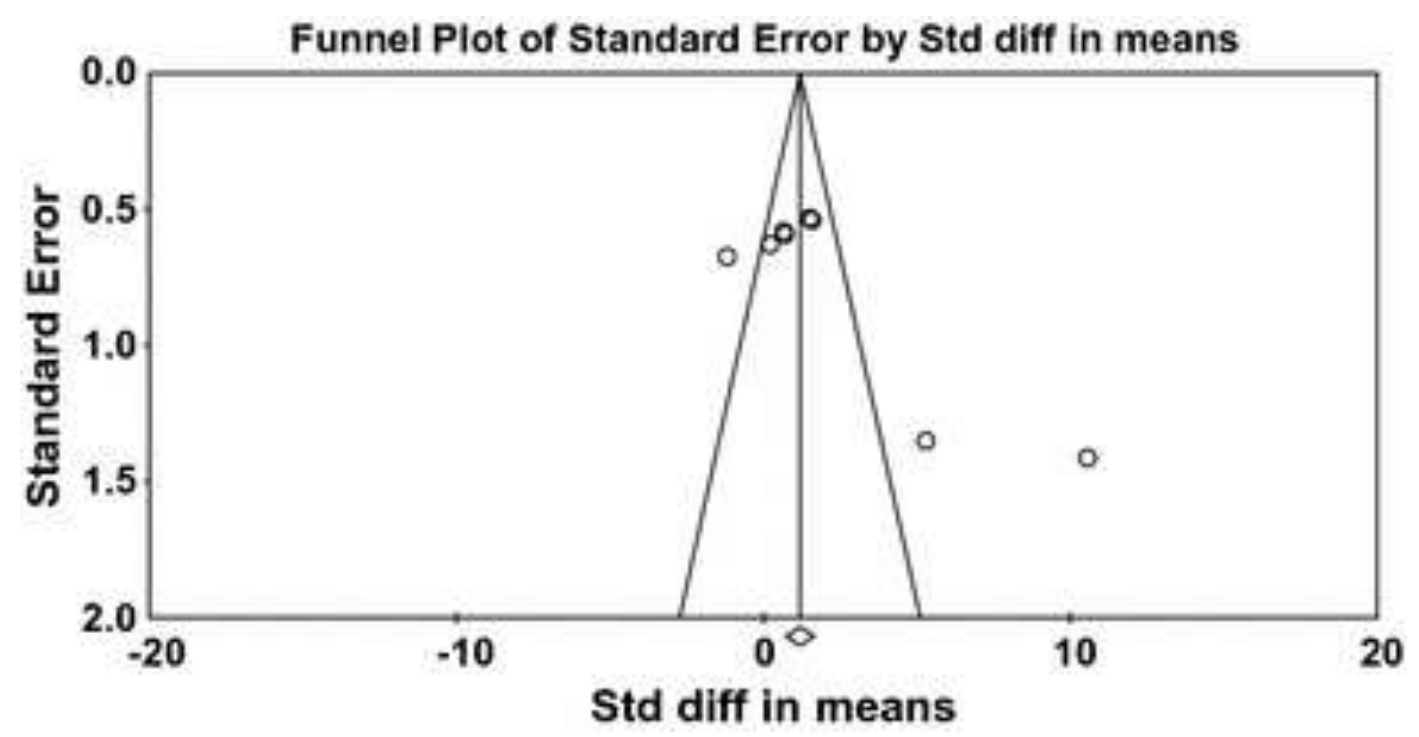

Figure 4: The asymmetrical funnel plot suggests publication bias confirmed by a significant Egger's intercept value of $\mathrm{p}=0.04$.

Using the GRADE quality assessment, the quality of evidence was double downgraded for the following two factors: imprecision of results with wide confidence intervals, and publication bias. This resulted in a low quality rating according to the GRADE criteria [14].

\section{Load to failure}

Load to failure was reported in all studies. The pooled estimate for all eight studies utilizing a random effect model demonstrated significant differences in the load to failure $(p=0.006)$ between single- and double-row repair techniques (SMD 1.228, 95\% CI: 0.055 to $5.226, \mathrm{p}=0.0001, \mathrm{I}_{2}=60.47 \%$ ). (Figure 5) 
Meta Analysis Load to Failure

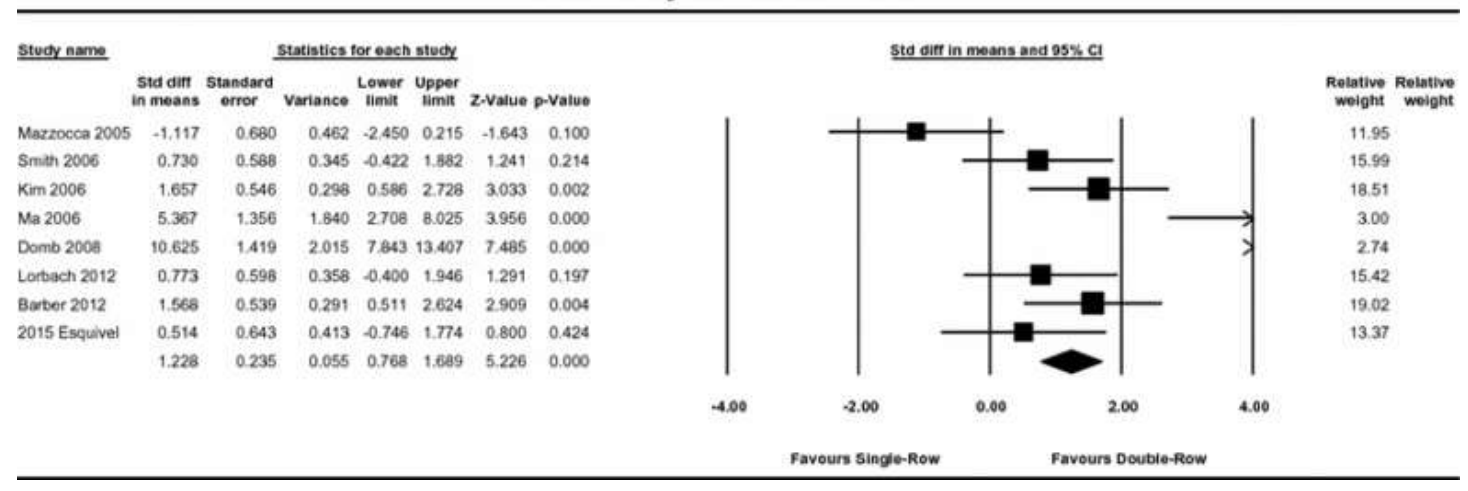

Figure 5: The pooled estimated for all studies suggested higher load to failure loads for double-row repairs.

\section{Failure Mode}

Lorbach, et al. was the only study to not report the failure mode (Table 2) [24]. The failure modes were divided into two groups. In group one, the suture was either failing at the anchor interface or the anchor was pulling out of bone. In group two, the suture failed at the tendon-suture interface by cutting through the tendon or by suture breakage. Statistical analysis using a $2 \times 2$ contingency table revealed that there was no significant difference between single- and double-row repairs in either of the two groups, respectively $(\chi=0.01, \mathrm{p}=0.92, \chi=0.02, \mathrm{p}=0.65)$.

\section{Elongation/Gap Formation}

Elongation or gap formation was also reported in all studies. The pooled estimate for all eight studies utilizing a random effect model demonstrated significant differences in gap size $(\mathrm{p}=0.006)$ between single- and double-row repair failures (SMD 0.783, 95\% CI: 0.169 to $1.398, \mathrm{p}=0.012, \mathrm{I}_{2}=58.8 \%$ ). (Figure 6) 


\section{Meta Analysis Gap Formation}

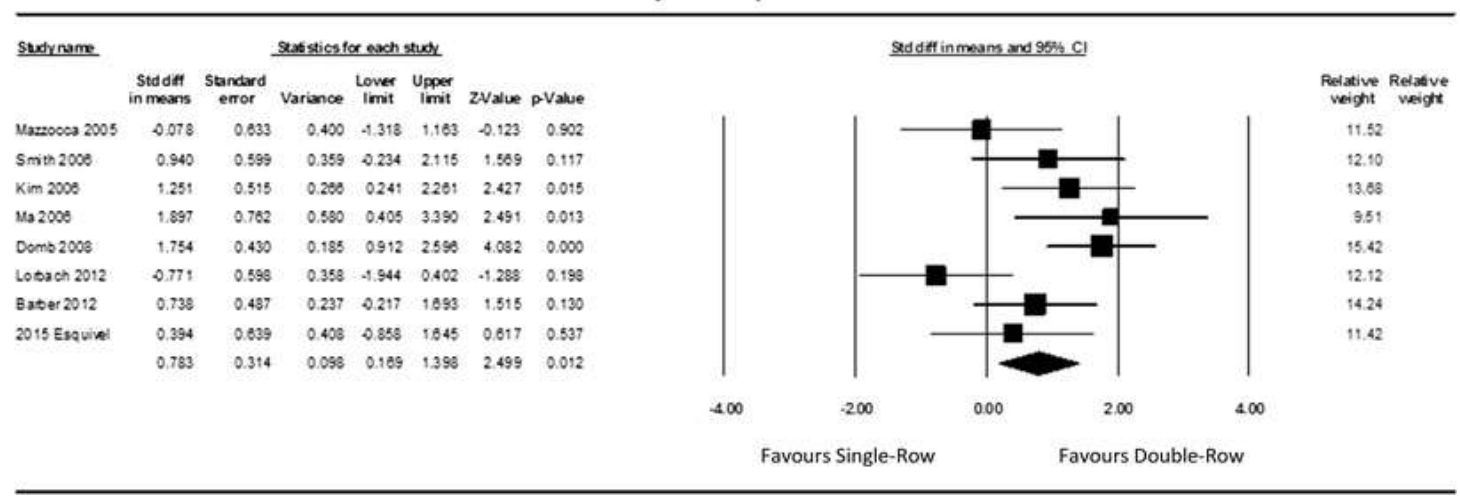

Figure 6: The pooled estimated for all studies from demonstrated smaller gap sizes for double-row repairs.

\section{Discussion}

The results of this meta-analysis of basic science studies comparing the biomechanical properties of single- versus double-row rotator cuff repair demonstrated that double-row repair resulted in significantly higher ultimate load to failure values and decreased elongation/gap formation. Failure modes between these two groups were not significantly different. To our knowledge, this is the first systematic review with meta-analysis comparing single - and double-row repair techniques with respect to the resulting biomechanical properties, such as load to failure, gap formation, and failure mode.

Studies have shown that stable fixation at the bone-tendon interface is one of the key factors in the early stages of healing [5]. Hughes \& An have calculated forces for the supraspinatus tendon during abduction, adduction, internal rotation, and external rotation [25]. They found that the calculated forces for maximal abduction exertion were $117 \mathrm{~N}$, for adduction $59 \mathrm{~N}$, and for maximal internal and external rotation, $115 \mathrm{~N}$ and $175 \mathrm{~N}$ respectively. Interestingly, the maximal load to failure in all eight included studies was above these calculated values for both single- and double row repair. 
These findings might explain the lack of any significant differences in clinical outcome scores between the two repair techniques [26]. In contrast, for larger tears above $3 \mathrm{~cm}$ double-row repair demonstrated superiority compared to the single-row technique [27]. This difference has been attributed to greater compression of the repair and a larger footprint area, which has been shown to increase the healing potential $[1,24]$. However, it is also possible that the absolute number of sutures, rather than the repair technique, is the common denominator for tendon healing. For instance, Jost et al. in an ovine rotator cuff model, demonstrated that a higher number of sutures decreased cyclic gap formation and increased load to failure [28]. However, single- and double-row repairs were biomechanically equivalent when the number of sutures was kept constant [28]. While the findings of this meta-analysis clearly favour double-row repair with regards to failure load, the testing methods of the included studies were heterogeneous and should not be generalized. In fact, the various methods utilized introduced bias, and these results should be interpreted with great caution.

Gap formation can potentially compromise tendon-bone healing, and was found to be one of the predictors for poor healing outcomes [1,29]. However, there is no clear consensus as to what is the exact value for gap formation or elongation to define a failure [29]. Several authors have suggested a gap of $5 \mathrm{~mm}$ as failure [30,31], whereas others have used values ranging anywhere from 3-10 $\mathrm{mm}$ [29]. Five of the eight studies that used single-row repair, and three of the eight studies using double-row repair technique [7,11,22-24], demonstrated values above the suggested threshold of 5 $\mathrm{mm}$. Interestingly, when using meta-analysis tools, double-row repair was superior to single-row repair with significantly lower gap formation. Similar phenomenon was 
also observed in load to failure, however, the testing protocols used were heterogeneous making comparisons between these studies difficult, and may have potentially introduced bias. Despite these significant findings, the results of these studies should be interpreted with caution. In addition, several authors have shown that the numbers of sutures passing through the tendon was the most important determinant of gap formation regardless of whether a single- or double-row repair was performed $[1,24,28]$. This suggests surgical technique was only one of the factors determining a successful outcome following surgery. Age, tissue quality, the size of the tear, retraction and muscle atrophy, and other patient related factors such as smoking, bone quality, chronic Vitamin D deficiency and diabetes were also shown to influence the healing outcome $[5,32]$.

Failure modes were not significantly different between the two groups and were equally distributed. For this measure, fifteen compared to thirteen specimens failed at the anchor-suture interface or via anchor pull-out, and 33 (69\% for single-row) compared to 36 (73\% for double-row) specimens failed within the suture-throughtendon mechanism. The majority of failures occurred at the suture-tendon interface, which has previously been reported as the weakest link of the reconstruction $[23,24]$. Increasing the number of sutures and the suture configuration, rather than the number of suture anchors, may strengthen rotator cuff repairs and obviate the need for doublerow repairs. However, if the number of sutures rather the number of anchors was the more important factor to reduce suture-tendon failure, the failure mode distribution should have been different between these two techniques. Unfortunately, the sample size of this study was insufficient to come to a valid conclusion (using G*Power 
3.1.9.2, Universität Düsseldorf, Germany), and the calculated post-hoc power was only 0.65 for failure mode.

The clinical relevance of this meta-analysis is unclear. In a recent meta-analysis investigating the clinical and radiological outcomes after arthroscopic single-row versus double-row repair Perser et al. demonstrated that double-row repair does not result in significant clinical improvement in both clinical and radiographic outcomes [33]. These findings were confirmed by Sheibani-Rad et al. and Dehaan et al demonstrating similar functional and clinical outcomes for both single-row and double row repairs [34,35]. Millet et al could not demonstrate any detectable differences between single-row and double-row repairs, but showed a significantly higher re-tear rate of single row (26\%) versus double-row (14\%) techniques [36]. However, patients who underwent double-row repair experienced significantly more pain with failure of repair [37].

Meta-analysis uses a quantitative approach to systematically assess the results of previous research as well as the results in a more precise estimate of the treatment effect [38]. The inclusion of non-randomized observational studies may be subject to sources of bias, and in fact may show larger treatment effects [39]. Pooling of these studies may also not lead to more definitive outcomes [38]. These are potential limitations with all non-randomized studies and this may also apply to our metaanalysis. Moreover, when using the GRADE quality assessment, the quality was double downgraded for wide confidence intervals and publication bias. In fact, there was a huge discrepancy for testing methods applied between the included publications. Cyclic loading protocols ranged from 50 to 3000 cycles, different cyclic 
loads and applied rates were used, and cadavers were tested at different humerus abduction angles. One study applied different loading protocols for single- and double-row testing, which the authors justified by using the tension differential between the normal anatomical footprint and medialized repairs, an argument that seems flawed [22]. Given these substantial differences, gap formation and elongation may be a direct result of rate of load rather than construct stability, and these values must be viewed with caution as outlined earlier.

For load to failure, the rate of displacement ranged from $1 \mathrm{~mm} / \mathrm{sec}$ to $33 \mathrm{~mm} / \mathrm{sec}$. Parimi, et al. showed that load to failure was rate dependent, with higher strain rates resulting in higher breaking strength [40]. However, a major limitation of Parimi, et al. was the large variance and confidence intervals with comparatively large loading rates. Loading rates below $33 \mathrm{~mm} / \mathrm{sec}$ are all considered slow, and one could assume that the results of load to failure testing would not be related to loading rate, and should therefore be a good estimate of failure with minimal bias [40].

Despite these inherent limitations, the analysis has confirmed superiority of the double-row repair with regards to load to failure. The lack of a uniform testing protocol is a major concern, and it is critical that the orthopaedic and biomechanical community develops and adheres to a single standard testing protocol to reduce confounding factors, which in turn would allow for more reliable comparisons between studies. For example, Burkhart et al. [30] suggested cyclic loading from 10$180 \mathrm{~N}$ at a rate of $33 \mathrm{~mm} / \mathrm{sec}$ based on the previous work of Ikai \& Fukunaga [36], where the load of $180 \mathrm{~N}$ represented approximately two thirds of the maximal contraction force that could be delivered [30,41]. Mazzocca, et al. suggested a load of 
$100 \mathrm{~N}$, which represents approximately $25 \%$ of the maximum rotator cuff contraction [10]. However, Mazzocca, et al. based these figures on loads that would occur during early rehabilitation with passive motion [10], whereas Burkart's parameters appear to simulate genuine clinical conditions more accurately [30]. There is currently no published evidence to best define the preferred loading conditions.

\section{Limitations}

As outlined earlier, the limitations of this meta-analysis are directly related to the limitations of the included studies. Double downgrading of study quality was necessary because of the inherent biases and study heterogeneity, substantially decreasing the external validity of both the included studies and this meta-analysis. Therefore, it is important to again emphasize these results should be interpreted with caution. The estimation of standard deviation as outlined by Hozo et al. could be seen as a limitation [20]. However, they demonstrated the estimation error is within $4 \%$ of the sample mean using this approach, suggesting its influence here would be insignificant [20]. Research theses and conference proceedings were excluded for this study, and the omission of these 'grey data' could have resulted in or contributed to publication bias. Publication bias was assessed with meta-analysis tools and was found to be present. However, the presence of publication bias is mainly caused by small sample size studies, the tendency of journals to reject negative studies, researchers deciding not to submit results, or sponsorships which prevent publication of non-favourable results [42]. 


\section{Conclusion}

The results of this systematic review and meta-analysis suggest that double-row repair is able to tolerate a significantly greater load to failure. Gap formation was also significantly lower in the double-row repair group.

\section{Compliance with ethical standards}

Not applicable

\section{Conflict of interest}

The authors declare no conflict of interest in relation to the presented body of research

\section{References}

[1] Roth, KM, Warth R, Lee JT, Millett PJ, ElAttrache NS. (2014) Arthroscopic single-row versus double-row repair for full-thickness posterosuperior rotator cuff tears. JBJS Reviews; 2 (7):e6. DOI:10.2106/JBJS.RVW.M.00081

[2] Lee TQ. (2013) Current biomechanical concepts for rotator cuff repair. Clin Orthop Surg; 5:89-97. DOI:10.4055/cios.2013.5.2.89

[3] Park MC, Cadet ER, Levine WN, Bigliani LU, Ahmad CS. (2005) Tendon-tobone pressure distributions at a repaired rotator cuff footprint using transosseous suture and suture anchor fixation techniques. Am J Sports Med;33(8):1154-9. DOI:10.1177/0363546504273053

[4] Ahmad CS, Stewart AM, Izquierdo R, Bigliani LU. (2005) Tendon-bone interface motion in transosseus suture and suture anchor rotator cuff repair techniques. Am J Sports Med; 33 (11):1667-1671. DOI:10.1177/0363546505278252

[5] Park JS, McGarry MH, Campbell ST, Seo HJ, Lee YS, Kim SH, et al. (2015) The optimum tension for briging sutures in transosseous-equivalent rotator cuff repair: A cadaveric biomechanical study. Am J Sports Med; 43 (9):2118-2125.

DOI:10.1177/0363546515590596

[6] Ahmad CS, Kleweno C, Jacir AM, Bell JE, Gardner TR, Levine WN, et al. (2008) Biomechanical performance of rotator cuff repairs with humeral rotation: a new rotator cuff repair failure model. Am J Sports Med; 36:888-892.

DOI:10.1177/0363546508316285 
[7] Esquivel AO, Duncan DD, Dobrasevic N, Marsh SM, Lemos SE. (2015) Load to failure and stiffness. Anchor placement and suture pattern effects on load to failure in rotator cuff repairs. Orthop J Sports Med; 7 (3): 2325967115579052. DOI:10.1177/2325967115579052

[8] Ma CB, Comerford L, Wilson J, Puttlitz CM. (2006) Biomechanical evaluation of arthroscopic rotator cuff repairs: double-row compared with single-row fixation. J Bone Joint Surg Am; 88:403-410. DOI:10.2106/JBJS.D.02887

[9] Galatz LM, Ball CM, Teefey SA, Middleton WD, Yamaguchi K. (2004) The outcome and repair integrity of completely arthroscopically repaired large and massive rotator cuff tears. J Bone Joint Surg Am; 86A:219-224

[10] Mazzocca AD, Millett PJ, Guanche CA, Santangelo SA, Arciero RA. ( 2005) Arthroscopic single-row versus double-row suture anchor rotator cuff repair. Am J Sports Med; 33 (12):1861-1868. DOI:10.1177/0363546505279575

[11] Smith CD, Alexander S, Hill AM, Huijsmans PE, Bull AMJ, Amis AA, et al. (2006) A biomechanical comparison of single and double-row fixation in arthroscopic rotator cuff repair. J Bone Joint Surg; 88A (11):2425-2431.

DOI:10.2106/JBJS.E.00697

[12] Mascarenhas R, Chalmers PN, Sayegh ET, Bhandari M, Verma NN, Cole B, et al. (2014) Is double-row rotator cuff repair clinically superior to single-row rotator cuff repair: a systematic review of overlapping meta-analyses. Arthroscopy; 30 (9):1156-1165. DOI:10.1016/j.arthro.2014.03.015

[13] Burks RT, Crim J, BrownN, Fink B, Greis PE. (2009) A prospective randomized clinical trial comparing arthroscopic single- and double row rotator cuff repair: magnetic resonance imaging and early clinical evaluation. Am J Sports Med; 37 (4):674-682. DOI:10.1177/0363546508328115

[14] Higgins JPT, Green S. (2011) Cochrane handbook for systematic reviews of interventions. Version 5.1.9 [updated March 2011]. The Cochrane Collaboration

[15] Moher D, Liberati A, Tetzlaff J, Altman DG. (2010) Preferred reporting items for systematic reviews and meta-analysis: the PRISMA statement. Int J Surg; 8:336-34. DOI:10.1016/j.ijsu.2010.02.007

[16] Park MC, ElAttrache NS, Tibone JE, Ahmad CS, Jun BJ, Lee TQ. (2007) Part I: Footprint contact characteristics for transosseous-equivalent rotator cuff repair technique compared with a double-row repair technique. J Shoulder Elbow Surg; 16 (4):461-468. DOI:10.1016/j.jse.2006.09.010

[17] Park MC, ElAttrache NS, Tibone JE, Ahmad CS, Jun BJ, Lee TQ. (2007) Part II: Biomechanical assessment for a footprint restoring transosseous-equivalent rotator cuff repair technique compared with a double-row repair technique. J Shoulder Elbow Surg; 16 (4):469-476. DOI:10.1016/j.jse.2006.09.011 
[18] Pietschmann MF, Hölzer A, Rösl C, Scharpf A, Niethammer T, Jansson , et al. (2010) What humeri are suitable for comparative testing of suture anchors? An ultrastructural bone analysis and biomechanical study of ovine, bovine and human humeri and four different anchor types. J Biomech; 43 (6):1125-1130.

DOI:10.1016/j.jbiomech.2009.12.021

[19] Hartling L, Hamm M, Milne A, Vandermeer B, Santaguida PL, Ansari M, et al. (2012) Validity and inter-rater reliability testing of quality assessment instruments. (Prepared by the University of Alberta Evidence-based Practice Center under Contract No. 290-2007-10021-I.) AHRQ Publication No. 12-EHC039-EF. Rockville, MD: Agency for Healthcare Research and Quality. March 2012. www.effectivehealthcare.ahrq.gov/reports/final.cfm. [accessed on 12 August 2016]

[20] Hozo SP, Djulbegovic B, Hozo I. (2005) Estimating the mean and variance from the median, range, and the size of a sample. BMC Med Res Methodol; 5:13. DOI:10.1186/1471-2288-5-13

[21] Barber FA, Drew OR. (2012) A biomechanical comparison of tendon-bone interface motion and cyclic loading between single-row, triple laoded cuff repairs and double-row, suture tape cuff repairs using biocomposite anchors. Arthroscopy; 28 (9):1197-1205. doi: 10.1016/j.arthro.2012.02.015

[22] Domb BG, Glousman RE, Brooks A, Hansen M, Lee TQ, ElAttrache NS. (2008) High-tension double-row footprint repair compared with reduced tension single-row repair for massive rotator cuff tears. J Bone Joint Surg; 90 Suppl 4:35-39.

DOI:10.2106/JBJS.H.00650

[23] Kim DH, ElAtrrache NS, Tibone JE, Jun BJ, DeLaMora SN, Kitne RS, et al. (2006) Biomechanical comparison of a single-row versus double-row suture anchor technique for rotator cuff repair. Am Sports Med; 34 (3):407-414.

DOI:10.1177/0363546505281238

[24] Lorbach O, Kieb M, Raber F, LC Busch, Kohn D, Pape D. (2012) Comparable biomechanical results for a modified single-row rotator cuff reconstruction using triple loaded suture anchors versus a sutre-bridging double-row repair. Arthroscopy; 28 (2):178-187. DOI:10.1016/j.arthro.2011.08.298

[25] Hughes RE, An KN. (1996) Force analysis of rotator cuff muscles. Clin Orth Relat Res; 330:75-83

[26] Millett PJ, Warth RJ, Dornan GJ, Lee JT, Spiegl UJ. (2014) Clinical and structural outcomes after arthroscopic single-row versus double-row rotator cuff repair: a systematic review and meta-analysis of level I randomized clinical trials. J Shoulder Elbow Surg; 23 (4):586-597. DOI:10.1016/j.jse.2013.10.006

[27] Zhang Q, Ge H, Zhou, Yuan C, Chen K, Cheng B. (2013) Single-row or doublerow fixation techniques for full thickness rotator cuff tears: a meta-analysis. PLOS One; 8 (7):e68515. DOI:10.1371/journal.pone.0068515 
[28] Jost PW, Khair MM, Chen DX, Wright TM, Kelly AM, Rodeo SA. (2012) Suture number determines strength of rotator cuff repair. J Bone Joint Surg Am; 94 (14); e100. DOI:10.2106/JBJS.K.00117

[29] Goschka AM, Hafer JS, Reynolds KA, Aberle II NS, Baldini TH, Hawkins MJ, et al. (2015) Biomechanical comparison of traditional anchors to all-suture anchors in a double-row rotator cuff repair cadaver model. Clinical Biomechanics; 30:808-813. DOI:10.1016/j.clinbiomech.2015.06.009

[30] Burkhart SS, Diaz Pagan JL, Wirth MA, Athanasiou KA. (1997) Cyclic loading of anchor-based rotator cuff repairs: confirmation of the tension overload phenomenon and comparison of suture anchor fixation with transosseous fixation. Arthroscopy; 13(6):720-724.

[31] Nelson CO, Sileo MJ, Grossman MG, Serra-Hsu F. (2008) Single-row modified mason-allen versus double-row arthroscopic rotator cuff repair: a biomechanical and surface area comparison. Arthroscopy; 24 (8):941-948.

DOI:10.1016/j.arthro.2008.03.011

[32] Abtahi AM, Granger EK, Tashjian RZ. (2015) Factors affecting healing after arthroscopic rotator cuff repair. World J Orthop; 6 (2):211-220.

DOI:10.5312/wjo.v6.i2.211

[33] Perser K, Godfrey D, Bisson L. (2011) Meta-analysis of clinical and radiographic outcomes after arthroscopic single-row versus double-row rotator cuff repair. (2011) Sports Health; 3 (3):268-274

[34] Sheibani-Rad S, Giveans MR, Arnoczky SP, Bedi A. (2013) Arthroscopic singlerow versus double-row repair: a meta-analysis of the randomized clinical trials.

Arthroscopy; 29 (2):343-348

[35] DeHaan AM, Axelrad TW, Kaye E, Silvestri L, Puskas B, Foster TE. (2012) Does double-row rotator cuff repair improve functional outcome of patients compared with single-row technique? A systematic review. Am J Sports Med; 40 (5):1176-1185

[36] Millett PJ, Warth RJ, Dornan GJ, Lee JT, Spiegl UJ. (2014) Clinical and structural outcomes after arthroscopic single-row versus double-row rotator cuff repair: A systematic review and meta-analysis of level I randomized clinical trials. J Shoulder Elbow Surg; 23 (4):586-597

[37] Yang J Jr, Robbins M, Reilly J, Maerz T, Anderson K. (2017) The clinical effect of rotator cuff repair: a meta-analysis of arthroscopic single-row and double-row repairs. Am J Sports Med; 45 (3):733-741

[38] Haidich AB. (2010) Meta-analysis in medical research. Hippokratia; 14 (Suppl 1):29-37

[39] Ioannidis JPA, Haidich A-B, Pappa M, Pantazis N, Kokori SI, Tektonidou MG, et al. (2001) Comparison of evidence of treatment effects in randomized and nonrandomized studies. JAMA;286: 821-830. 
[40] Parimi M, Zhao C, Thoreson AR, An KN, Amadio PC. (2012) Does velocity affect failure strength after tendon repair? J Biomech; 45 (16):2939-2942.

DOI:10.1016/j.jbiomech.2012.08.039

[41] Ikai M, Fukunaga T. (1968) Calculation of muscle strength per unit of crosssectional area of human muscle. Int Zeitschrift Angewandte Physiol einschliesslich Arbeitsphysiol; 26:26-31.

[42] Thornton A, Lee P. (2000) Publication bias in meta-analysis: its causes and consequences. J Clin Epidemiology; 53:207-216 